\title{
Flu Near You: An Online Self-reported Influenza Surveillance System in the USA
}

\author{
Rumi Chunara*1, 2, Susan Aman², Mark Smolinski ${ }^{3}$ and John S. Brownstein ${ }^{1,2}$ \\ ${ }^{1}$ Harvard Medical School, Boston, MA, USA; 'BBoston Children's Hospital, Boston, MA, USA; ${ }^{3}$ Skoll Global Threats Fund, San \\ Francisco, CA, USA
}

\section{Objective}

To develop a participatory system for monitoring the activity of influenza-like-illness among the United States general population.

\section{Introduction}

The emergence of new influenza strains including H1N1, H5N1, $\mathrm{H} 3 \mathrm{~N} 2 \mathrm{v}$ as well as other respiratory pathogens such as SARS, along with generally weak information about household and community transmission of influenza, enforce the need for augmented influenza surveillance. At the same time, Internet penetration and access has grown, with $82 \%$ of American adults using the Internet [1], enabling transfer and communication of information that can be collected and aggregated in near real-time. Surveillance targeted towards influenza in other countries, and towards malaria in India, has previously been executed with good user engagement [2,3]. In this study, we created an online participatory influenza surveillance tool in the United States, called Flu Near You.

\section{Methods}

Volunteer users were primarily solicited via collaboration with the American Public Health Association and their members' networks starting Oct. 24, 2011. Upon registration, each user is sent a weekly email, taking them to the Flu Near You website. On the website they fill in a short survey asking if they had any of 10 symptoms: fever, cough, sore throat, shortness of breath, chills/night sweats, fatigue, nausea or vomiting, diarrhea, body aches and headache, in the last week. Users can also enroll their household members and enter information in for them weekly. A map of influenza activity is made available to users, and anyone accessing the website [Figure 1]. On the map, the number of individuals reporting with no symptoms, some symptoms, or Influenza-like illness are visualized, aggregated to the zip code level. Users can also compare the contributed data with other surveillance systems: the Centers for Disease Control and Prevention, and Google Flu Trends for the same time period [Figure 1]. We also obtained user feedback through a survey in early July 2012.

\section{Results}

As of August 21, 2012, there are over 9300 Flu Near You users, from all 50 states. $94.0 \%$ of users are between 20 and 70 years, although $37.2 \%$ of household members are $<20$ years old. Overall $62.0 \%$ of members (users and household) were female. We found that $46.4 \%$ of users answered 3 or more surveys. Qualitatively from survey responses, we learned that simple feedback and an emphasis on public health education are important in this type of system.

\section{Conclusions}

We found that it is possible to engage users in a symptom self-reporting system and augment information about influenza for the na- tion. Increased uptake would increase the value of the system for the public and public health professionals. Flu Near You is expanding in its second season, working to increase user participation. Other connected projects are also examining the expansion into other diseases with high prevalence and in need of augmented surveillance. With a larger user base and through a longer period of execution, systems like Flu Near You will help to improve our understanding of influenza epidemiology as well as guide implementation of relevant and timely public health interventions, for example in estimating vaccination rates and efficacy among different demographic groups. Information reported by individuals can augment traditional public health surveillance methods for more timely detection of disease outbreaks, monitoring disease activity and increasing the public's engagement in their own and population's health.

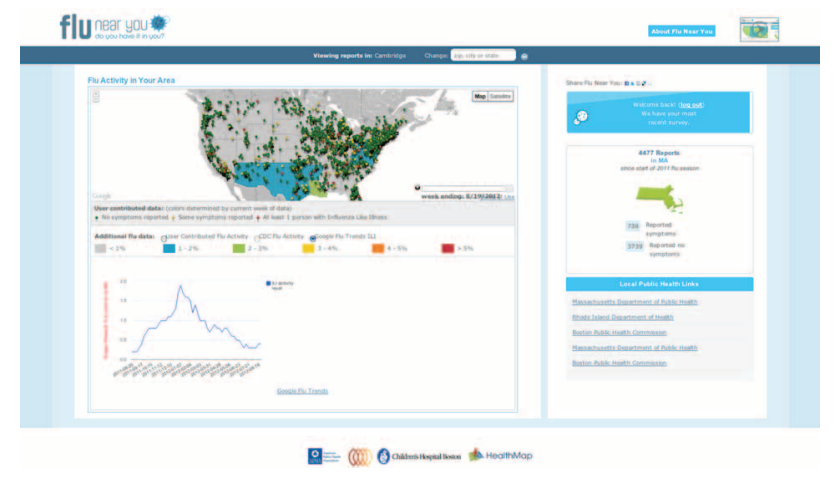

Keywords

influenza; surveillance; crowdsourcing

\section{Acknowledgments}

All of the Flu Near You users who have contributed information.

\section{References}

[1] Pew Internet \& Aerican Life Project. Available at: pewinternet.org. Accessed Aug. 22, 2012.

[2] Marquet RL, et al. Internet-based monitoring of influenza-like illness (ILI) in the general population of the Netherlands during the 20032004 influenza season. BMC Public Health. 2006; 6: 242.

[3] Chunara R, et al. (2012) Online reporting for malaria surveillance using micro-monetary incentives, in urban India 2010-2011. Malaria J 11: 43.

\section{*Rumi Chunara}

E-mail: rumi@alum.mit.edu 